\title{
Manejo del paciente con COVID-19 en etapa neonatal y embarazo
}

\author{
Management of the COVID-19 patient in neonatal and pregnancy
}

Elsa Gabriela Saucedo Rodríguez,* Rosangela Del Razo Rodríguez, ${ }^{\ddagger}$ Deneb Algedi Morales Barquet ${ }^{\S}$

\footnotetext{
* Pediatra. Residente de Neumología Pediátrica, INER.

₹ Neumólogo y Broncoscopista Pediatra. Médico adscrito a Neumología Pediátrica, INER.

§ Neonatólogo Intensivista. Centro Médico ABC Santa Fe.
}

La neumonía viral es una causa importante de morbimortalidad en el embarazo, ya que está asociada con eventos adversos, tales como ruptura prematura de membranas, parto pretérmino o restricción del crecimiento intrauterino. La información disponible sobre el SARS-CoV-2 durante el embarazo es limitada, por lo que se ha tomado en cuenta la información relacionada con otros coronavirus altamente patogénicos como el SARS o MERS, ${ }^{1}$ pero en el caso del SARS-CoV-2 es dudosa la transmisión vertical. ${ }^{2}$

Según los datos existentes en muestras que fueron sometidas a pruebas de SARS-CoV-2 de líquido amniótico, sangre del cordón umbilical, placenta y muestras de leche materna de recién nacidos de madres infectadas, todas éstas resultaron negativas en la detección del virus. ${ }^{3}$

Las mujeres embarazadas deben seguir las medidas generales para detener la propagación del SARS-CoV-2, esto es: cubrir la boca al toser, evitar contacto con personas enfermas y lavarse frecuentemente las manos con agua y jabón o aseo de manos con desinfectante de manos a base de alcohol. ${ }^{4}$

Durante el desarrollo del SARS en el 2003, se administró ribavirina a mujeres embarazadas, pero se presentaron efectos teratogénicos, por lo que no hay un tratamiento actual recomendado, a causa de este potencial efecto. ${ }^{5}$

Financiamiento: Ninguno.

Conflicto de intereses: Ninguno.

Rev Latin Infect Pediatr 2020; 33 (s1): s70-s74

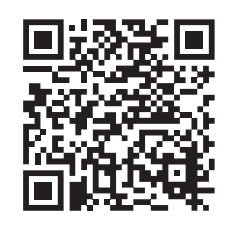

\section{ESTADO ACTUAL DE LA INFECCIÓN POR SARS-COV-2 EN MUJERES EMBARAZADAS Y NEONATOS}

En un estudio prospectivo del Hospital de Zhongnan de la Universidad de Wuhan, China, del 1 al 31 de enero de 2020, se estudiaron a nueve pacientes atendidas por cesárea con diagnóstico de COVID19; siete de las madres presentaron fiebre y dos sufrimiento fetal. Todas las muestras estudiadas de líquido amniótico, cordón umbilical y leche materna resultaron negativas, y ninguno de los recién nacidos desarrolló neumonía o murió..$^{1,3}$

En la misma provincia de China, se estudiaron a 33 recién nacidos de madres con SARS-CoV-2; tres de ellos se encontraron con datos clínicos y radiológicos de neumonía; un paciente presentó hisopado nasofaríngeo y anal positivo para SARSCoV-2; otro presentó leucocitosis, linfopenia, elevación $\mathrm{CPK}-\mathrm{MB}$, radiografía de tórax con neumonía e hisopado nasofaríngeo y anal positivos. El tercero presentó síndrome de distrés neonatal con hisopados nasofaríngeo y anal positivos al diagnóstico, que negativizaron a los siete días. ${ }^{5}$

La infección neonatal por los virus respiratorios podría ocurrir después del nacimiento por medio de la inhalación de aerosoles producidos por la tos de la madre, de los trabajadores de la salud o de otras
Citar como: Saucedo REG, Del Razo RR, Morales BDA. Manejo del paciente con COVID-19 en etapa neonatal y embarazo. Rev Latin Infect Pediatr. 2020; 33 (s1): s70-s74. https://dx.doi.org/10.35366/96672 
Rev Latin Infect Pediatr 2020; 33 (s1): s70-s74

fuentes en un ambiente hospitalario. Basándonos en la experiencia pasada de mujeres embarazadas que desarrollaron MERS-CoV y SARS-CoV no se ha confirmado alguna transmisión intrauterina de la madre al feto. ${ }^{3}$

\section{CUADRO CLÍNICO EN EL RECIÉN NACIDO}

El periodo de incubación es de 1 a 14 días con una media estimada de 5.2 días. En $97.5 \%$ de los casos, los síntomas se desarrollan en los primeros 10 días. El cuadro clínico puede oscilar desde asintomático hasta distrés respiratorio. Éste último se presenta más frecuentemente con alguna enfermedad subyacente, tal como cardiopatía e hidronefrosis. ${ }^{4}$

Los síntomas más comunes son fiebre, fatiga durante la alimentación y tos seca. Puede haber síntomas de la vía respiratoria superior como congestión nasal, descarga retronasal, síntomas gastrointestinales como vómito, diarrea y se pueden presentar taquipnea y fiebre, sin embargo, en las series se refiere que, en general, los pacientes se encuentran estables. ${ }^{2}$

El diagnóstico, en términos de gravedad, debe enfocarse en confirmar o descartar oportunamente neumonía y síndrome de insuficiencia respiratoria aguda por COVID-19. ${ }^{6}$ Para la población pediátrica menor a dos meses (incluyendo la neonatal), se debe descartar la presencia de neumonía, la cual puede iniciar con taquipnea $>60 \mathrm{rpm}$ y al menos uno de los siguientes síntomas:

1) Cianosis central o $\mathrm{SpO}_{2}<92 \%$.

2) Otros síntomas: tos, apneas, dificultad respiratoria grave con quejido, estertores y/o tiraje intercostal. Estos síntomas pueden ocurrir conforme la condición clínica progresa o empeora y, usualmente, se presenta una semana después del inicio de la enfermedad.

El diagnóstico se basa en el antecedente de contacto y se realiza a través de la detección del virus por RT-PCR en muestras de hisopados nasofaríngeos (detección positiva en menos del 50\%), secreciones de tracto respiratorio inferior (con un rango elevado de sensibilidad, pero debe individualizarse por el riesgo de infecciones cruzadas), en sangre y en heces. ${ }^{2}$ Es importante considerar la coinfección con Mycoplasma, VSR, influenza A o B y virus Epstein-Barr. ${ }^{2,7}$
En la población neonatal no está descrita la estratificación de la enfermedad con apoyo de tomografía pulmonar. Debemos minimizar los riesgos de trasladar al paciente para evitar la contaminación de los equipos de radiología y la diseminación hospitalaria del virus, por lo que se puede evaluar la realización seriada de ultrasonido pulmonar en el sitio de atención (point of care ultrasound) para detectar y dar seguimiento en caso de progresión y/o la presencia de complicaciones. ${ }^{8}$

\section{TRATAMIENTO NEONATAL}

Si la madre del neonato tiene un resultado positivo para SARS-CoV-2 debe aislarse en una habitación con presión negativa. El tratamiento sólo es de soporte, como oxígeno, mantenimiento electrolítico y de equilibrio ácido-base. En el caso de los recién nacidos con distrés respiratorio grave, el tratamiento se basa en la administración de dosis altas de surfactante pulmonar, óxido nítrico inhalado, ventilación de alta frecuencia, remdesivir, IFN $\alpha 2 b$ nebulizado (usado en MERS-CoV y SARS-CoV) y la consideración de oxigenación por membrana extracorpórea (ECMO) en caso de contar con ello. ${ }^{9,10}$

\section{CUIDADOS DEL RECIÉN NACIDO CON \\ MADRE POSITIVA PARA SARS-COV-2}

Aunque el ambiente ideal de un recién nacido es con la madre, se recomendó la separación temporal de un recién nacido y su madre enferma durante la pandemia de $\mathrm{H} 1 \mathrm{~N} 1$, lo cual se considera prudente; también se debe plantear el aislamiento de otros recién nacidos sospechosos o contagiados en una habitación con presión negativa, y monitorizar la temperatura al menos durante 10 días. . $^{411}$

La OMS recomienda mantener el amamantamiento tanto en casos de madres confirmadas, como probables, siempre y cuando se mantengan las medidas de prevención de infección por microorganismos transmitidos por gotas y por contacto. En casos de madres con enfermedad grave, se puede recurrir a la extracción de la leche, sin embargo, el tipo de alimentación del recién nacido deberá consensuarse entre la paciente y el equipo tratante, y entre el estado de salud de la madre y el recién nacido. ${ }^{12}$

Las visitas deben ser restringidas; ${ }^{13}$ en caso de que la madre se encuentre infectada con COVID-19 se debe mantener separada hasta que: 
a) Se encuentre afebril por 72 horas sin el uso de antipiréticos.

b) Tenga mejoría de los síntomas respiratorios.

c) Obtenga resultados negativos de dos muestras consecutivas para SARS-CoV-2 de hisopado nasofaríngeo tomadas con 24 horas de diferencia.

Otros autores refieren que los padres con resultados positivos de COVID-19 no deben visitar a los recién nacidos hasta siete días después del inicio de la enfermedad y hasta que se encuentren asintomáticos.

\section{REANIMACIÓN NEONATAL}

En cuanto a las consideraciones neonatales y materno infantiles, éstas tienen relevancia imprescindible, ya que las mujeres embarazadas y sus fetos representan una población de alto riesgo. ${ }^{14}$ Si bien, en algunos reportes se ha documentado que hay enfermedad desde las primeras horas de vida, ${ }^{15,16}$ aún existen dudas sobre la viabilidad de transmisión del neonato al personal de salud tratante. ${ }^{14,16,17}$

Los cambios cardiovasculares, pulmonares y fisiológicos de la madre incrementan el riesgo de

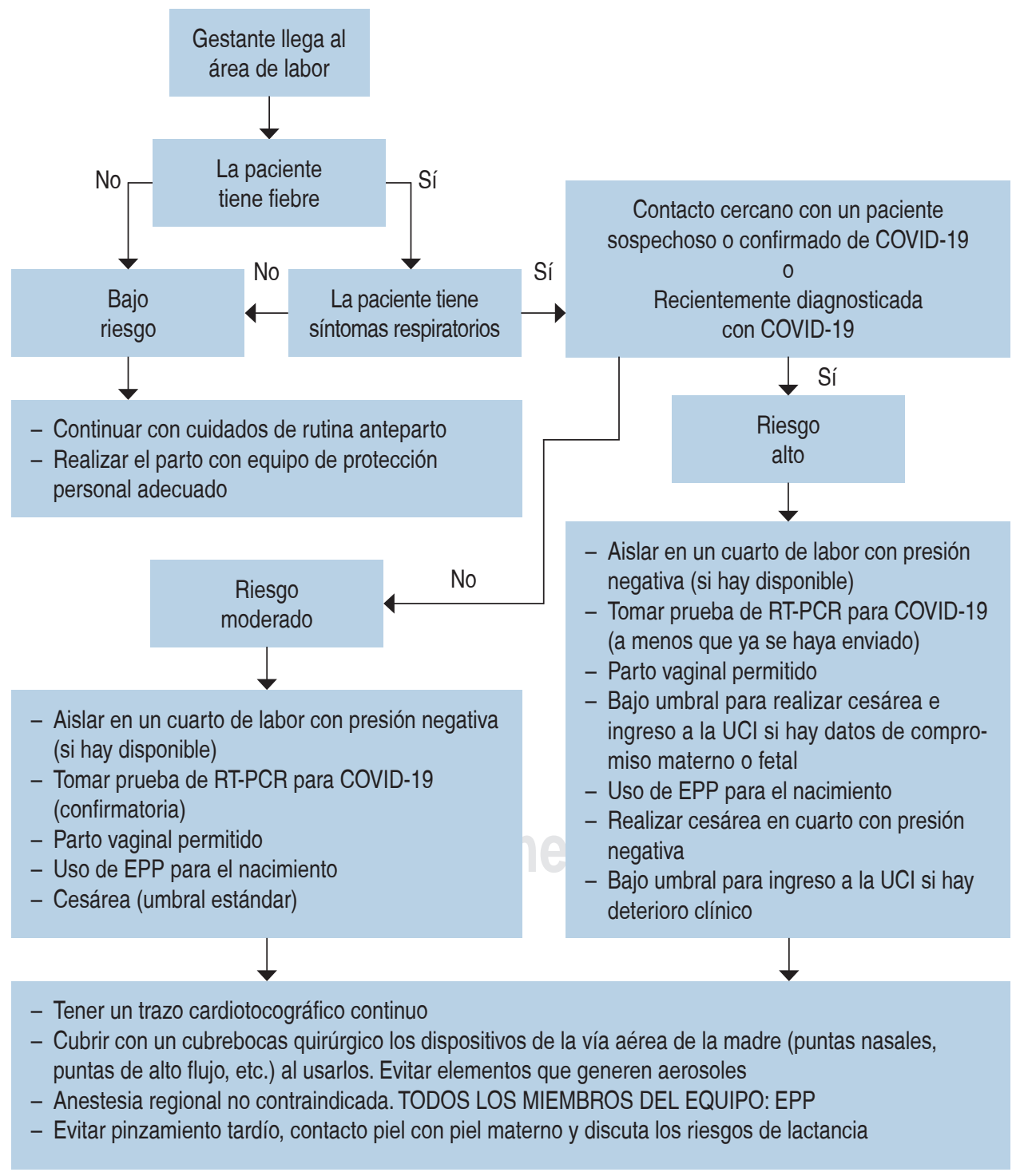

Figura 1:

Propuesta para el manejo integral al atender a la gestante y la reanimación del recién nacido. 
descompensación en el binomio. ${ }^{14,17}$ La preparación para el momento del parto debe tener las mismas consideraciones básicas que para recibir a un neonato hijo de madre no sospechosa o confirmada de COVID-19; sin embargo, debe implementarse el protocolo de colocación del EPP en el personal de salud ${ }^{16-18} y$, además, colocar una mascarilla quirúrgica en la madre, con la finalidad de minimizar el riesgo de exposición a aerosoles. ${ }^{18}$

No hay recomendaciones concluyentes para realizar una cesárea que reduzca la transmisión de madre a hijo. En las epidemias de SARS-CoV y MERS-CoV no se documentó transmisión, ${ }^{18,19}$ sin embargo, los reportes actuales aún son muy escasos para determinar una posición objetiva. ${ }^{14,17,18}$ La postura en cuanto al contacto piel con piel del neonato asimismo es controversial, si bien algunas lo recomiendan si el binomio está estable, ${ }^{16}$ otras, al no garantizar que exista o no una transmisión por leche materna, lo desaconsejan. ${ }^{18}$

En cuanto al manejo de pacientes prematuros y la administración de esteroides antenatales, las diferentes guías ${ }^{14,18}$ no los desaconsejan, pero recomiendan una vigilancia estrecha de la madre y el producto, de acuerdo con lo que se ha visto con otros virus que se presentan en la gestante, tal como es el VIH y otros coronavirus. ${ }^{18,19}$

Las recomendaciones generales para la atención del recién nacido son las mismas que las que dispone la AHA con la AAP, haciendo hincapié en la colocación del EPP. ${ }^{17}$ Si bien los pasos iniciales no son EGA, la inminente administración de medicamentos endotraqueales en el contexto de una reanimación neonatal avanzada y la succión de secreciones, así como la aplicación de surfactante pulmonar, sí lo son, por lo que resulta imprescindible utilizar este tipo de equipo. Una última recomendación se hace al momento del transporte del neonato, con medidas de aislamiento estricto para evitar la transmisión al personal de salud, madre y al resto de familiares. ${ }^{17,18}$ La Figura 1 resume la propuesta del manejo integral para la atención de la gestante y la reanimación del recién nacido. ${ }^{13,16,18}$

\section{CONCLUSIONES}

Hay un conocimiento limitado respecto a las infecciones por coronavirus durante el embarazo, y la mayor parte del conocimiento ha sido resultado del estudio epidemiológico de dos diferentes enfermedades: SARS y MERS. Este coronavirus puede causar muerte materna en un número pequeño pero significativo.
El coronavirus podría resultar en efectos adversos para el feto como restricción del crecimiento intrauterino, nacimiento pretérmino, aborto espontaneo y muerte perinatal. No se han documentado casos de transmisión vertical con SARS o MERS. Las mujeres embarazadas deben considerarse de alto riesgo para desarrollar infección grave por SARS-CoV-2, ${ }^{1,3}$ aunque los reportes de casos en neonatos son pocos y se refieren como pacientes estables.

\section{REFERENCIAS}

1. Karimi-Zarchi M, Neamatzadeh H, Dastgheib SA, Abbasi $\mathrm{H}$, Mirjalili SR, Behforouz A et al. Vertical transmission of coronavirus disease 19 (COVID-19) from infected pregnant mothers to neonates: a review. Fetal Pediatr Pathol [Internet]. 2020; 39 (3): 246-250. Disponible en: https://europepmc.org/backend/ptpmcrender. fcgi?accid=PMC7157948\&blobtype $=$ pdf

2. Lu Q, Shi Y. Coronavirus disease (COVID-19) and neonate: what neonatologist need to know. J Med Virol [Internet]. 2020; 92 (6): 1-4. Disponible en: https://onlinelibrary.wiley. com/doi/pdf/10.1002/jmv.25740

3. Chen H, Guo J, Wang C, Luo F, Yu X, Zhang W et al. Clinical characteristics and intrauterine vertical transmission potential of COVID-19 infection in nine pregnant women: a retrospective review of medical records. Lancet. 2020; 395 (10226): 809-815.

4. Organización Mundial de la Salud (OMS). Coronavirus disease 2019 (COVID-19) information for clinicians caring for children and pregnant women [Internet]. Situation Report-66. 2020. Disponible en: https://www.who.int/docs/ default-source/coronaviruse/situation-reports/20200326sitrep-66-covid-19.pdf?sfvrsn=9e5b8b48_2

5. Zeng L, Xia S, Yuan W, Yan K, Xiao F, Shäo J et al. Neonatal early-onset infection with SARS-CoV-2 in 33 neonates born to mothers with COVID-19 in Wuhan, China. JAMA Pediatr [Internet]. 2020; 23 (77): E1-E3. Disponible en: https:// scholar.google.com/scholar_url?url=https://jamanetwork. com/journals/jamapediatrics/articlepdf/2763787/ jamapediatrics_zeng_2020_ld_200013.pdf\&hl=es\&sa=T\&oi =ucasa \&ct=ufr\&ei=15kMX5uSM5KiywTkjav4DQ\&scisig=AA GBfm3ux3ewNhgcFtcD6-Vyzt0pI6IOLQ

6. Coria-Lorenzo JJ, Calva-Rodríguez RG, Unda-Gómez JJ, Martínez-Núñez JG, García-Carrillo LE, Neme-Díaz GA et al. Consenso sobre la infección por COVID-19 (SARSCoV-2). Rev Enferm Infecc Pediatr [Internet]. 2020; 32 (132): 1656-91. Disponible en: https://biblat.unam.mx/hevila/ Revistadeenfermedadesinfecciosasenpediatria/2020/vol32/ no132/1.pdf

7. Liu Y, Chen H, Tang K, Guo Y. Clinical manifestations and outcome of SARS-CoV-2 infection during pregnancy. J Infect [Internet]. 2020. Disponible en: https://doi.org/10.1016/j. jinf.2020.02.028

8. Buonsenso D, Pata D, Chiaretti A. COVID-19 outbreak: less stethoscope, more ultrasound. Lancet Respir Med [Internet]. 2020; 8 (5): e27. Disponible en: https://www.ncbi.nlm.nih. gov/pmc/articles/PMC7104316/pdf/main.pdf

9. Wang L, Shi Y, Xiao T, Fu J, Feng X, Mu D et al. Chinese expert consensus on the perinatal and neonatal management for the prevention and control of the 2019 
novel coronavirus infection (First edition). Ann Transl Med. 2020; 8 (3): 47.

10. Maxwell C, McGeer A, Tai KFY, Sermer M; Maternal Fetal Medicine Committee; Infectious Disease Committee. Management guidelines for obstetric patients and neonates born to mothers with suspected or probable severe acute respiratory syndrome (SARS). J Obstet Gynaecol Can. 2009; 31 (4): 358-364.

11. Panahi L, Amiri M, Pouy S. Risks of novel coronavirus disease (COVID-19) in pregnancy; a narrative review. Arch Acad Emerg Med. 2020; 8 (1): e34.

12. Schwartz DA, Graham AL. Potential maternal and infant outcomes from (Wuhan) coronavirus 2019-nCoV infecting pregnant women: lessons from SARS, MERS, and other human coronavirus infections. Viruses. 2020; 12 (2): 194.

13. Puopolo KM, Hudak ML, Kimberlin DW, Cummings J. Management of infants born to mothers with COVID-19. Initial guidance [internet]. American Academy of Pediatrics Committee on Fetus and Newborn, Section on Neonatal Perinatal Medicine, and Committee on Infectious Diseases. 2020. Disponible en: https://downloads.aap.org/AAP/PDF/ COVID 19 Initial Newborn Guidance.pdf

14. Dashraath P, Wong JLJ, Lim MXK, Lim LM, Li S, Biswas A et al. Coronavirus disease 2019 (COVID-19) pandemic and pregnancy. Am J Obstet Gynecol. 2020; 222 (6): 521-531.

15. Yang Y, Peng F, Wang R, Guan K, Jiang T, Xu G et al. The deadly coronaviruses: the 2003 SARS pandemic and the 2020 novel coronavirus epidemic in China. J Autoimmun [Internet]. 2020; 109: 102434. Disponible en: eader. elsevier.com/reader/sd/pii/S0896841120300470?token=73 5B919C89A22A3CC2AE2E6EBA1C0C26031534F38E2B8
9FD993DBCDC4D6CE97260918DA55339BD5491BDAC5 A56FD839D

16. Resuscitation Council (UK). Resuscitation Council UK Statements on COVID-19 (Coronavirus), CPR and Resuscitation [Internet]. Resuscitation Council (UK). 2020. Disponible en: https://www.resus.org.uk/sites/default/ files/2020-06/Resuscitation Council UK Statement on COVID-19 in relation to CPR and resuscitation in first aid and community settings 13052020 .pdf

17. Edelson DP, Sasson C, Chan PS, Atkins DL, Aziz K, Becker LB et al. Interim guidance for basic and advanced life support in adults, children, and neonates with suspected or confirmed COVID-19. Circulation [Internet]. 2020; 141 (25): e933-e943. Disponible en: https://www.ahajournals.org/doi/ epub/10.1161/CIRCULATIONAHA.120.047463

18. Chandrasekharan $P$, Vento $M$, Trevisanuto $D$, Partridge $E$, Underwood MA, Wiedeman $\mathrm{J}$ et al. Neonatal resuscitation and postresuscitation care of infants born to mothers with suspected or confirmed SARS-CoV-2 Infection. Am J Perinatol. 2020; 37 (8): 813-824.

19. Mullins E, Evans D, Viner RM, O'Brien P, Morris E. Coronavirus in pregnancy and delivery: rapid review. Ultrasound Obstet Gynecol [Internet]. 2020; 55 (5): 586592. Disponible en: https://obgyn.onlinelibrary.wiley. com/doi/pdf/10.1002/uog.22014\%4010.1002/\%28IS SN\%291469-0705.covid-19_in_obgyn

Correspondencia:

Dra. Rosangela Del Razo Rodríguez

E-mail: rosangelarr@yahoo.com 